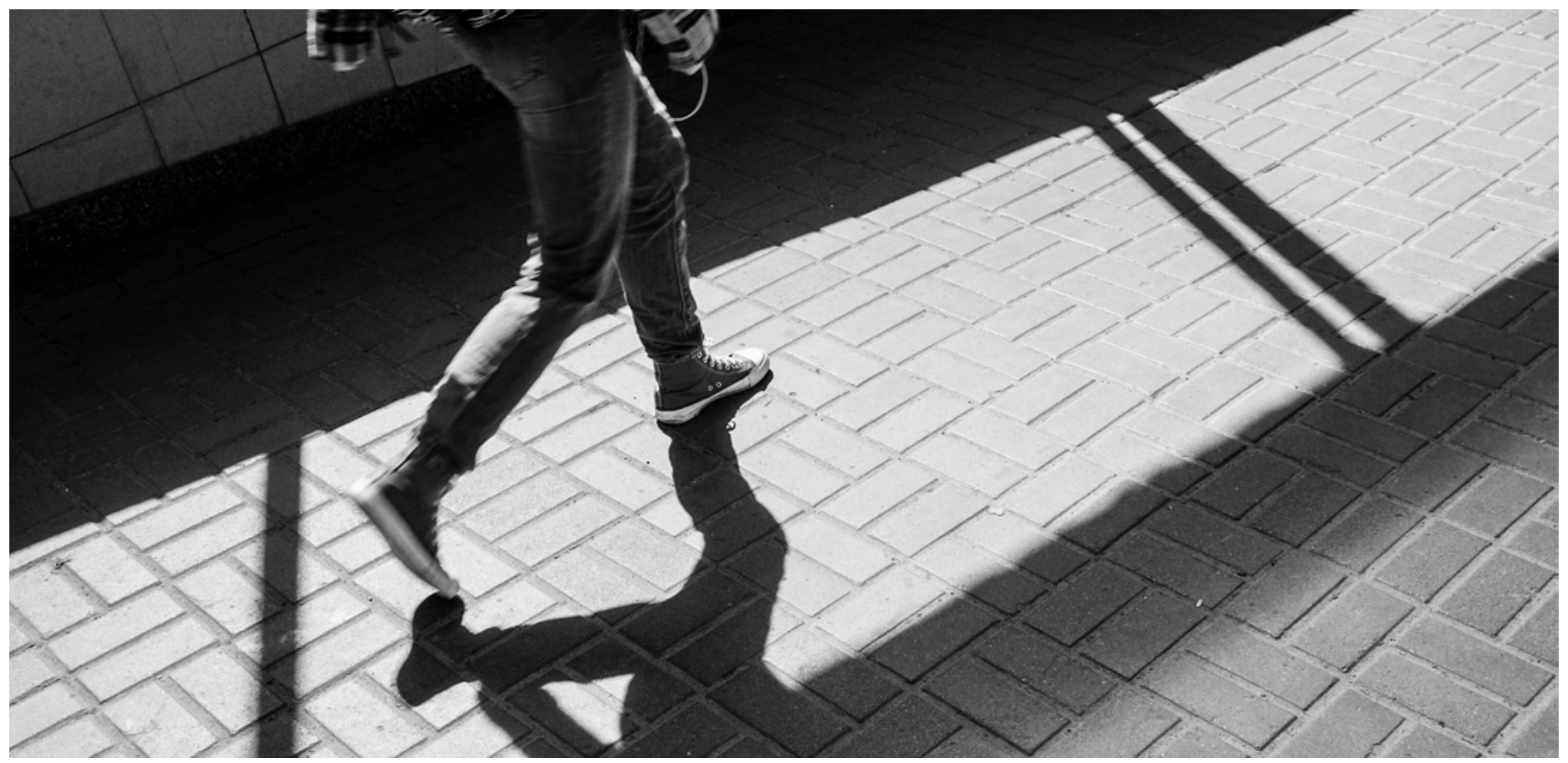

\title{
Gesundheitsversorgung für Sans Papiers
}

\section{Marianne Jossen}

M.A. Soziologie / Master Public Health

Welche Erfahrungen machen Patienten, Patientinnen und Fachleute mit der Gesundheitsversorgung für Sans Papiers? Wer nachfragt, taucht ein in eine von Abhängigkeiten und Fragilität, aber auch von Mut und starken Persönlichkeiten geprägte Welt.

Am 12. Juni 2018 debattierte der Nationalrat zur Frage der Sans Papiers [1]; Menschen, die sich in der Schweiz aufhalten, ohne eine legale Bewilligung dazu zu haben. In einer Motion hatte die Kommission für soziale

\section{Résumé}

Quelles sont les expériences des patientes et des patients ainsi que des professionnels avec l'assistance médicale pour les sans-papiers? Lorsque I'on s'interroge à ce sujet, on découvre un univers marqué par les dépendances et la fragilité, mais aussi par le courage et des personnalités fortes. En dépit du droit à une caisse-maladie garantie par le droit, la situation des sans-papiers en Suisse reste précaire s'agissant des soins. Dans ce contexte, les aspects administratifs et financiers sont essentiels.
Sicherheit und Gesundheit verlangt, Sans Papiers von den Sozialversicherungen - zum Beispiel der Krankenversicherung - auszuschliessen.

Die staatspolitische Kommission hatte darauf reagiert: Das sei vorschnell und es brauche zuerst eine Abklärung, was denn die Konsequenzen einer solchen radikalen Aberkennung wären. Zudem, so die staatspolitische Kommission, sollen die Erfahrungen mit der Legalisierung von Härtefällen, die im Rahmen der «Operation Papyrus» in Genf gesammelt wurden, einfliessen. Die Kommission beantragte ein Postulat, also eine genauere Abklärung in einem Bericht. Der Bundesrat unterstützte dieses Vorgehen, die Kommission für soziale Sicherheit und Gesundheit wiederum zog ihre Motion zurück; nicht ohne Raunen aus der rechten Ecke. 
Sozialversicherungen für Sans Papiers? Das Bundesamt für Sozialversicherungen bestätigte 2002 das Recht und die Pflicht der Sans Papiers, eine Krankenversicherung abzuschliessen [2]. Seit 2012 müssen auch Menschen, die von der Nothilfe leben - auch sie gelten als Sans Papiers - von den Kantonen versichert werden.

Und wie sieht es in der Praxis aus? Wie achten Sans Papiers auf ihre Gesundheit? Und welchen Beitrag leisten Fachleute? Während eines Zeitraums von rund anderthalb Jahren habe ich mit Sans Papiers und Fachpersonen über dieses Thema diskutiert, nach Antworten auf diese Fragen gesucht und von ihnen gelernt.

\section{Schmerzen? Dafalgan. Ärztliche Beglei- tung? Fehlanzeige}

Gleich zu Beginn: Die überwiegende Mehrheit der Sans Papiers hat keine Krankenversicherung abgeschlossen. Wir kommen später darauf zurück. Die Feststellung erlaubt, die Frage zu stellen, welche Konsequenzen eine Aberkennung des Rechts auf Krankenversicherung für Sans Papiers hätte.

\section{NGOs bauen Netzwerke aus engagierten Gesundheitsfachleuten auf, die für reduzierte Tarife an Randzeiten das Nötigste tun.}

Ein erster Reflex wäre anzunehmen, dass Sans Papiers - wie andere Menschen ohne Versicherung - die Behandlungspflicht öffentlicher Spitäler im Notfall beanspruchen können; einmal davon abgesehen, dass die Definition eines Notfalls und das genaue Vorgehen nicht immer klar ist. Gerade für Sans Papiers. Sie nehmen häufig an, dass sie auf dem Notfall irgendwann doch einen Pass zeigen müssen. Sie sind sich unsicher, was passiert, wenn sie die Behandlung nicht bezahlen können. Eine Betreibung? Ein Albtraumszenario für Sans Papiers, die dann befürchten müssen, ausgeschafft zu werden.

Vertrauen haben Menschen ohne legalen Aufenthalt und ohne Versicherung viel eher in eine der NGOs, die sich auf die Gesundheitsanliegen von Sans Papiers spezialisiert haben. Deren gibt es rund ein Dutzend in der Schweiz [3]. Gelangt ein Sans Papiers mit einem gesundheitlichen Anliegen an eine solche NGO, setzt sich ein fragiler Prozess in Gang. Kann das Problem vor Ort behoben werden? Hat die NGO die nötigen Ressourcen? Das Personal ist spezialisiert darin, auch komplexe Anliegen aufzunehmen, und kennt sich aus in der Welt der Sans Papiers. Oftmals machen Patienten hier erstmals die Erfahrung einer Gesundheitsversorgung, die nicht von Fragmentierung, $\mathrm{Ab}$ - bruch und Diskontinuität geprägt ist, wie die Sans Papiers das etwa oftmals im Asylprozess erleben. Benötigt ein Anliegen jedoch mehr als Gehör, Medikation und vielleicht eine basale Untersuchung, muss der Patient weitergewiesen werden. NGOs bauen Netzwerke aus engagierten Gesundheitsfachleuten auf, die für reduzierte Tarife an Randzeiten das Nötigste tun. Abhängigkeit von Goodwill und Grossherzigkeit wird so teilweise von den Sans Papiers zu den NGOs transferiert, die ihrerseits zu Bittstellern werden, wo keine Rechte eingefordert werden können.

Und was tun die, die nicht von einem Bekannten oder Freund von einer solchen NGO gehört haben? Sie tun, was sie können, um ihre Handlungsfähigkeit bezüglich ihrer Gesundheit aufrechtzuerhalten. Dabei geraten sie oft in Situationen, so paradox das klingt, in denen sie sich selber marginalisieren und ihrer Gesundheit schaden müssen, um diese zumindest notdürftig zu erhalten. Medikamente, die ein Rezept benötigen, kann man sich in der einen oder anderen Apotheke erbitten; sofern das Personal bereit ist, die eigenen professionellen Regeln zu umgehen. Ärztliche Begleitung? Kein Thema. Schmerzen? Dafalgan. Bluthochdruck? Ignorieren. Diabetes? Viel laufen, damit der Zucker runtergeht, sich dafür aber eine Wunde am Fuss einhandeln.

\section{Teuer bezahlte Autonomie}

Warum haben aber nun so wenige Sans Papiers eine Versicherung? Nun, der Abschluss - sofern man überhaupt um sein Recht weiss - ist eine administrative Kunst für sich. Zunächst braucht man einen vertrauenswürdigen Menschen mit legalem Aufenthalt, der Postadresse und Bankkonto zur Verfügung stellt. Versicherungen sind zwar verpflichtet, Sans Papiers aufzunehmen, manche winden sich aber trotzdem, nutzen legale und administrative Grauzonen. Versicherungen, die offener sind, kooperieren teilweise mit

\section{Versicherungen sind zwar verpflichtet, Sans} Papiers aufzunehmen, manche nutzen aber legale und administrative Grauzonen.

den NGOs, die auch beim Versicherungsabschluss helfen, wollen aber gleichzeitig nicht zu attraktiv werden für eine Klientel, von der sie eher Schwierigkeiten und Kosten als glatte administrative Abläufe erwarten. Anstatt gemeinsam dafür zu sorgen, dass alle Menschen in der Schweiz versichert sind, finden sich NGOs und Versicherungen wieder in kräftezehrenden Verhandlungen darüber, ob man nicht den einen oder anderen Patienten bei einem anderen Anbieter unterbringen könnte. 
Neben diesen administrativen Schwierigkeiten sind die Versicherungskosten der wesentliche Grund dafür, dass Sans Papiers ohne Schutz bleiben. Ein Sans Papiers verdient in der Schweiz monatlich schätzungsweise zwischen 600 und 2000 Schweizer Franken. Wohlgemerkt ohne jegliche Sozialbeiträge, ohne Unfall- oder Kündigungsschutz und ohne jegliche Garantie auf Regelmässigkeit. Der Abschluss einer Versiche-

\section{Ist die Versicherung einmal abgeschlossen, bedeutet sie einen enormen Zugewinn an Autonomie und Sicherheit.}

rung kann schnell in finanzielle Abhängigkeiten treiben; und sich gegen Ausbeutung zu wehren wird noch schwieriger. Nichtsdestotrotz: Wer Menschen begeistert über eine Versicherung reden hören möchte, selbst wenn sie ihnen bis zur Hälfte ihres Einkommens abverlangt, der sollte sich mit einem Sans Papiers zusammensetzen. Ist die Versicherung einmal abgeschlossen, bedeutet sie einen enormen Zugewinn an Autonomie und Sicherheit. Aber selbst mit gültiger Versicherung ist ein Besuch im Spital für eine Sans Papiers nicht ohne Hürden. Noch immer gibt es Krankenkassen, die Sans Papiers keine Krankenversicherungskarte ausstellen. Spitaladministrationen, getrieben von politisch gewollten betriebswirtschaftlichen Logiken, reagieren mit Misstrauen, wenn der Versicherungsstatus nicht mithilfe der Karte verifiziert werden kann. Man ist gehalten, Profit zu machen. NGOs stellen Kostengarantien aus und schicken Freiwillige als Begleitung mit. Anstatt dass ein Dialog entsteht, der Gesundheitsversorgung für notabene versicherte Personen ermöglicht, geraten NGOs und Spitaladministrationen in unnötige Konfliktsituationen.

\section{Gesundheit für Sans Papiers}

Was ist nun mit der Versorgungssituation selbst, wenn einmal der Weg dorthin gefunden ist? Um es noch einmal in Erinnerung zu rufen: Die medizinische Versorgung von Sans Papiers bringt für die Fachpersonen keinerlei rechtliche Schwierigkeiten mit sich. Für viele Anliegen, gerade wenn es um gut isolierbare körperliche Beschwerden geht, braucht es keinerlei spezifische
Kompetenzen seitens medizinischer Praktikerinnen und Praktiker; zumal einige Sans Papiers, die schon über viele Jahre in der Schweiz leben, auch sprachlich keine Hürden haben. Anders sieht es aus, wenn es um Grundversorgung und um eine umfassendere Gesundheitsversorgung geht. Hier gilt es, Vertrauen aufzubauen, Zeit zu haben, damit der Patient auch über seine Situation als Sans Papiers sprechen kann. Die legale Situation als Sans Papiers ist eine Lebenssituation, die alle anderen Aspekte einer Persönlichkeit auszulöschen droht; eine Situation, die eine ungewöhnliche Belastung darstellt und ungewöhnliche Resilienz verlangt. Praktikerinnen und Praktiker der Medizin können hier Situationen schaffen, in denen Patienten Anliegen und Situationen diskutieren können, über die sie sich sonst weitgehend ausschweigen müssen.

Die legale Situation als Sans Papiers ist eine Lebenssituation, die alle anderen Aspekte einer Persönlichkeit auszulöschen droht.

Die Frage nach den Sozialversicherungen für Menschen ohne legalen Aufenthalt ist mit vielen Paradoxa behaftet. Sie ist geprägt von dem grundlegenden Widerspruch, der so bestimmend ist für Sans Papiers: Da sein und nicht da sein dürfen. Diese Widersprüche, gesellschaftlich geschaffen, brennen sich als Verletzungen in konkrete menschliche Körper ein.

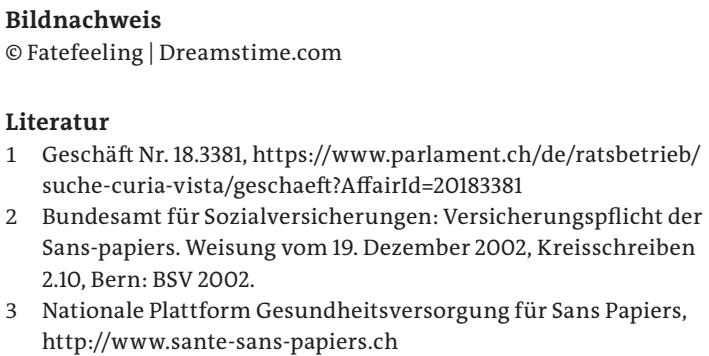
Sans-papiers. Weisung vom 19. Dezember 2002, Kreisschreiben 10, Bern: BSV 2002.

3 Nationale Plattform Gesundheitsversorgung für Sans Papiers, http://www.sante-sans-papiers.ch

Korrespondenz: Marianne Jossen M.A./Mph, 0795084317 marianne.jossen[at] icloud.com
Dieser Artikel basiert auf einer qualitativen Studie der Autorin zur Gesundheitsversorgung von Sans Papiers in einer Schweizer Region. Die Arbeit ist beim Verlag Open Book Publishers erschienen, steht dort gratis zum Download bereit und kann als Buch bestellt werden: https://www.openbookpublishers.com/ product/748. 